\title{
InSAR phase analysis: Phase unwrapping for noisy SAR interferograms
}

\author{
Riadh ABDELFATTAH ${ }^{1,2}$ \\ ${ }^{1}$ École Supérieure des Communications de Tunis (URISA/Sup'Com) \\ Tunisia \\ ${ }^{2}$ ITI/Telecom Bretagne \\ France
}

\section{Introduction}

Interferometric Synthetic Aperture Radar (InSAR) exploits the phase difference between two complex radar signals for extracting information about the earth surface. Some significant application fields concerned by InSAR techniques are Digital Elevation Model (DEM) generation (Graham, 1974), geophysical hazard analysis (Massonet \& Feigl, 1998), desertification (Bodart et al., 2009), deforestation (Balzter, 2001), glacier velocity measurements (Mattar et al., 1998) and land use classification (Wegmullerl \& Werner, 1997). They exploit the interferometric phase, which is proportional to the difference of the path lengths between the radar positions and a target on the earth surface (Gabriel \& Goldstein, 1988), and the interferometric correlation (Abdelfattah \& Nicolas, 2006), which depends on target characteristics (Abdelfattah \& Nicolas, 2002).

The phase of complex InSAR pair, called the interferogram, may be used in the reconstructing of high resolution terrain digital elevation models (DEM), in measuring earth displacement and many other topographic applications. However, before the reconstruction applications, the interferometric phase have to be unwrapped. the phase that is measured (observed) is only modulo $2 \pi$, the so called principal phase value or wrapped phase. Moreover, it is well known that the speckle effects in the InSAR data generate noise in the InSAR phase, which is also noisy due to various decorrelation effects (thermal noise, temporal, geometrical, etc.) between the two images used in the interferogram generation. The phase unwrapping consists in estimating the true regular phase profile (multiples of $2 \pi$ must be added or substracted) from the observed interferogram, which is noisy and wrapped, to make the phase image as smooth as possible. Formally, we have for each pixel $(i, j)$ :

$$
\phi_{i j}=\varphi_{i j}+2 k \pi
$$

where $\phi_{i j}$ is the true phase value (unwrapped phase), $\varphi_{i j}$ is the observed phase (wrapped), and $k \in \mathbb{Z}$ is an integer accounting for the number of $2 \pi$ multiplies (Ghiglia \& Pritt, 1998). Generally, the phase noise have to be reduced from the wrapped phase before the computation of the exact multiplies number. This will produce a regular fringe patterns and reducing the number of irregularities (phase discontinuities). Thus, the phase unwrapping step would be easier after the filtering step. However, the filtering process may cause a loss of resolution, 
which will affect the accuracy of the extracted information. Moreover, areas with high coherence don't need really to be filtered. So, the interferogram global filtering should be avoided, and an adaptive filtering approach should be considered.

The phase unwrapping process have to respect the trade-off between the spatial resolution and the accuracy of the interferometric phase. Two groups of phase unwrapping algorithms have been proposed in the literature: the first group uses local approach (Goldstein et al., 1988 ) and the second one uses global approach (Ghiglia \& Romero, 1994).

- The local approaches perform the summation of the phase differences along a selected path from pixel to pixel across the interferogram. They are path dependent and requires the phase residues connection using a phase residue map which could be directly obtained from the interferogram. A phase residue represents a potential origin of phase unwrapping errors. These approaches perform well in areas with sufficient high coherence. However, they fail when the interferogram is noisy due to the residues created by the noise.

- The global approaches are path independent and referred to as the least squares method (Ghiglia \& Romero, 1994). The unwrapped phase is then obtained as the minimizer, in the mean-square sense, of a dependent (on unwrapped phase and phase difference fields) functional. These approaches are powerful in the local reconstruction of the unwrapped phase, but propagates the unwrapping errors from decorellated parts of the image into high coherent areas.

In the literature, it is shown that these methods are optimized when the extraction of the true phase values is performed on a filtered interferogram. Unfortunately, noise reduction is achieved at the expense of a loss in spatial resolution. Moreover, these approaches are incapable to recover inconsistencies due to the InSAR acquisition system: they cannot extract the phase profile across regions of invalid information (such as shadow or layover regions).

In this chapter, we propose a modified filtering algorithm to the López and Fabregas (LópezMartínez \& Fabregas, 2002) noise reduction algorithm for the interferometric phase noise in SAR interferometry using a multiresolution approach. Our contribution to the existing algorithm consists on the exploitation of the InSAR coherence map in order to generate a more adaptive mask for each decomposition level. Moreover, an improved Markov Random Field (MRF) path independent phase unwrapping method is proposed. It is based on the Rodriguez and Servin (Rodriguez-Vera \& Servin, 1995) framework which is well adapted for phase unwrapping of locally inconsistent and noisy principal value phase fields that may contain regions of invalid information. This framework is based on Bayesian estimation theory with the use of Markov random field models to construct the prior distribution, so that the solution to the unwrapping problem is characterized as the minimizer of a piecewise-quadratic functional. Our contribution is then the definition of an adaptive (and more realistic) energy functional for the optimization of the random field.

In the next section, we will introduce the InSAR phase characteristics: it includes the statistics, the decorellation effects and the noise model. In the third section, InSAR phase noise reduction using the multiresolution approach is presented and validated through simulated and real interferograms. The InSAR phase unwrapping based on MRF model is detailed in the forth section. 


\section{InSAR phase characteristics}

The InSAR phase is affected by the presence of several decorrelation effects, which collectively result in an interferogram phase noise.

\subsection{InSAR phase statistics}

It is known that, under the traditional assumptions of fully developed speckle, single look complex SAR images follow the "circular Gaussian model", i.e., the phase follows a uniform law between 0 and $2 \pi$, and the real and the imaginary intensities follow Gamma laws with the same variance. An interferometric pair is composed of two single look complex SAR images: each pixel is represented by a vector $\mathbf{Z}$ of dimension 2 whose components $z_{1}$ and $z_{2}$ are the values of pixel on each image of the interferometric pair, where each component follows the circular Gaussian model. Then, the circular Gaussian model with 2 dimensions applies to this vector $\mathbf{Z}$, and its probability density function (pdf) is given by (Maître, 2001):

$$
p_{z}(\mathbf{Z})=\frac{1}{\pi^{2} \operatorname{det}\left(\mathbf{C}_{\mathbf{z}}\right)} \exp \left(-\mathbf{Z}^{* T} \mathbf{C}_{\mathbf{z}}^{-\mathbf{1}} \mathbf{z}\right)
$$

where $\mathbf{C}_{\mathbf{z}}$ is the covariance matrix of $\mathbf{Z},{ }^{*}$ the complex conjugate operator and ${ }^{T}$ signifies hermetian transpose. With the assumption that in each of the correlated signals, $z_{1}$ and $z_{2}$, the quadrature components of each sample are zero-mean Gaussian processes and independently distributed, the complex Wishart distribution for the covariance matrix estimate can be derived (Wishart, 1928). The covariance matrix is given by:

$$
\mathbf{C}_{\mathbf{z}}=\left(\begin{array}{cc}
\sigma_{1}^{2} & \sigma_{1} \sigma_{2} D e^{j \beta} \\
\sigma_{1} \sigma_{2} D e^{-j \beta} & \sigma_{2}^{2}
\end{array}\right)
$$

where $D$ and $\beta$ are the coherence magnitude and the interferometric phase. $\sigma_{1}^{2}$ and $\sigma_{2}^{2}$ are the individual signal variances. An estimate of $\mathbf{C}_{\mathbf{z}}$ could be calculated by the $N$-look sample covariance matrix, as the average of several independent samples, and it is given as (Touzi \& Lopez, 1996)

$$
\widehat{\mathbf{C}}_{\mathbf{z}}=\frac{1}{N} \sum_{k=1}^{N} \mathbf{Z}_{k}{ }^{T} \mathbf{Z}_{k}^{*}=\frac{1}{N} \sum_{k=1}^{N}\left[\begin{array}{cc}
z_{1}^{2}(k) & z_{1}(k) z_{2}^{*}(k) \\
z_{1}^{*}(k) z_{2}(k) & z_{2}^{2}(k)
\end{array}\right]
$$

where $N$ is the number of looks. Thus, the off-diagonal elements describe the complex multilook interferogram, where the noise problem is reduced by averaging neighboring pixels. Integrating the complex Wishart-distribution with respect to the diagonal elements will leads to the joint density function of the interferometric magnitude and phase given by (full expressions are given in (Touzi \& Lopez, 1996)). Integrating this joint pdf with respect to the magnitude leads to the marginal phase density function (Lee et al., 1994):

$$
p^{\prime}(\varphi)=\frac{\Gamma\left(N+\frac{1}{2}\right)\left(1-d^{2}\right)^{N} d \cos (\varphi-\beta)}{2 \sqrt{\pi} \Gamma(N)\left(1-d^{2} \cos ^{2}(\varphi-\beta)\right)^{N+\frac{1}{2}}}+\frac{\left(1-d^{2}\right)^{N}}{2 \pi}{ }_{2} F_{1}\left(N, 1 ; \frac{1}{2} ; d^{2} \cos ^{2}(\varphi-\beta)\right)
$$

An example of the marginal pdf (3) is plotted in Fig. 1 for $N=9$ and different coherence values, varying between 0.9 and 0.3 . The peak of the distribution is located at $\varphi=\beta$ which is considered to be nul in the example of Fig. 1. It can be shown that the phase is uniformly 
distributed when $D=0$ and becomes a Dirac delta function when $D=1$. It is also obvious that multi-look processing improves the phase accuracy, which lead to the decreasing of the phase variance for a larger number of looks (Lee et al., 1994).

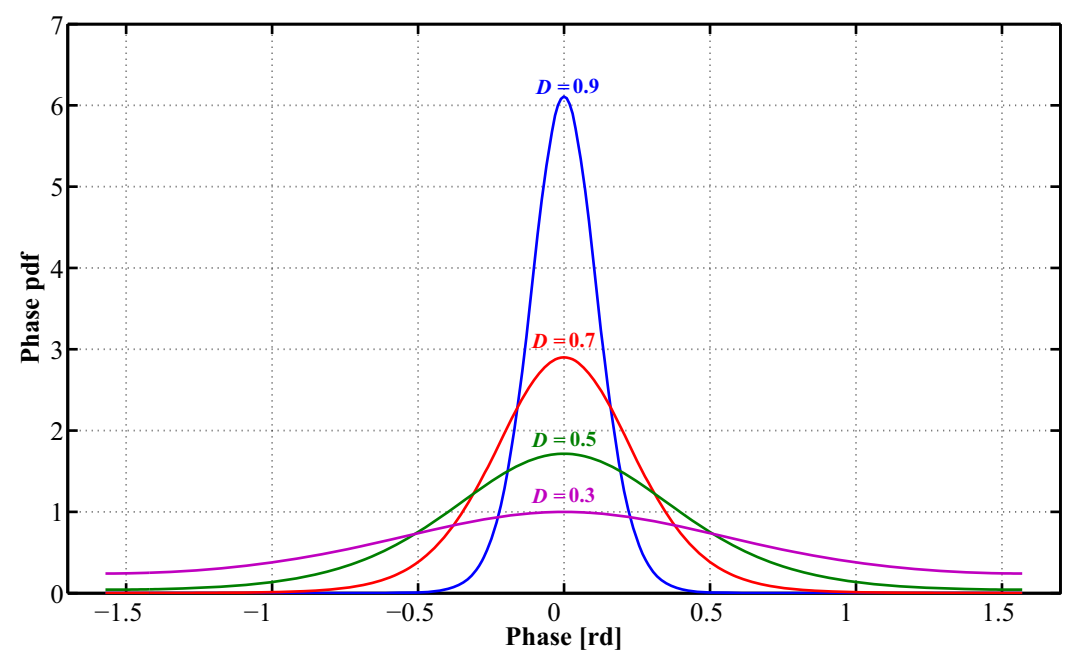

Fig. 1. Theoretical marginal multi-look pdf of the interferometric phase with $N=9$ and $\beta=0$.

\subsection{Decorrelation effects}

The interferometric phase can be affected by mainly three decorrelation factors: thermal $\left(\hat{\rho}_{\text {thermal }}\right)$, temporal $\left(\hat{\rho}_{\text {temporal }}\right)$ and geometrical $\left(\hat{\rho}_{\text {geom }}\right)$ decorrelation (Hanssen, 2001). The Doppler centroid decorrelation and the processing induced decorrelation could be avoided or neglected (Franceschetti \& Lanari, 1999). The considered decorrelation effects increase the interferometric phase noise, which will complicate more the unwrapping phase operation. When considered together, the above decorrelation factors are multiplicative so the approximate total decorrelation value $\hat{\rho}_{\text {total }}$ can be estimated as (Zebker \& Villasenor, 1992):

where :

$$
\hat{\rho}_{\text {total }} \simeq \hat{\rho}_{\text {thermal }} \cdot \hat{\rho}_{\text {temporal }} \cdot \hat{\rho}_{\text {geom }}
$$

- $\hat{\rho}_{\text {thermal }}$ is the decorrelation value induced by the temperature of the sensors (thermal noise) on the interferometric phase during acquisition. It can be expressed by the signal to noise (SNR) of the specific sensor by (Zebker \& Villasenor, 1992):

$$
\hat{\rho}_{\text {thermal }}=\frac{1}{1+S N R^{-1}}
$$

The value of the $S N R$ ratio is defined as:

$$
S N R=\frac{P_{S}}{P_{N}}
$$


where $P_{S}$ is the signal power and $P_{N}$ the noise power.

- $\hat{\rho}_{\text {temporal }}$ is the temporal decorrelation which indicates all the physical changes occurring on the surface between acquisitions, mainly in the case of repeat-pass interferometry. It includes changes of soil moisture content, surface roughness and vegetation. An analytical model of the temporal decorrelation is given in (Zebker \& Villasenor, 1992) by:

$$
\hat{\rho}_{\text {temporal }}=\exp \left(-\frac{1}{2}\left(\frac{4 \pi}{\lambda}\right)^{2}\left(\sigma_{x}^{2} \sin ^{2} \theta+\sigma_{z}^{2} \cos ^{2} \theta\right)\right)
$$

where $\sigma_{x}$ and $\sigma_{z}$ are the average standard deviation, respectively, in azimuth and in range of a random displacement of the reflectors inside a resolution cell. $\lambda$ is the radar wavelength and $\theta$ is its average look angle.

- $\hat{\rho}_{\text {geom }}$ represents the effects of the geometric decorrelation on the interferometric phase due to the interferometric acquisition geometry (baseline, registration, target rotation with respect to the sight line). Indeed, the same ground resolution cell is imaged from two slightly different looking angle. This means that the sensors (for both the interferometric acquisitions) don't look at the target with the same incidence angle. The numerical assessments of these effects depend on the length of the baseline, the incidence angle and the spatial resolution. In (Zebker \& Villasenor, 1992), the authors provide the geometric baseline decorrelation function as the result of the phase offset due to the difference in the incidence angle between the two InSAR acquisitions:

$$
\hat{\rho}_{\text {geom }}=1-\frac{2 B_{\perp} l_{g} \cos \theta}{\lambda R_{0}}
$$

where $B_{\perp}$ is the orthogonal component of the baseline to the radar look direction, $l_{g}$ the ground range resolution, and $R_{0}$ the slant range. A modified version of (7), given by Lee and Liu (Lee \& Liu, 1999), includes the terrain slope $\alpha$ is given by:

$$
\hat{\rho}_{\text {geom }}=1-\frac{c B_{\perp}\left|\cos \left(\theta_{0}-\alpha\right)\right|}{\lambda R_{0} B_{w}}
$$

where $c$ is the speed of light, $\theta_{0}$ the nominal incidence angle on the ellipsoidal earth $\left(23^{\circ}\right.$ for ERS-1 and ERS-2) and $B_{w}$ the frequency bandwidth of the transmitted signal.

\subsection{InSAR phase noise model}

The InSAR phase quality is measured with the absolute coherence (Abdelfattah \& Nicolas, 2006). Coherence interference due to reflection by random scatterers degrades the complex image. The amplitude is corrupted by multiplicative noise, while the phase is corrupted by additive noise. Indeed, based on (3), Lee et al. proved in (Lee et al., 1998) that: Since $\varphi$ distribution is symmetrical about $\beta, \beta$ is the mean. The standard deviation is independent of $\beta$. Consequently, $\varphi$ can be characterized by an additive noise model in the real domain:

$$
\varphi_{z}=\varphi_{x}+v
$$

where $\varphi_{z}$ is the measured value, $\varphi_{x}$ is the original phase to be estimated and $v$ is the zero-mean noise with the standard deviation $\sigma_{v}$. In the real domain, this phase noise model presents the problem of phase jumps. It is due to the complex representation of the interferometric phase 
(a wrapped phase, modulo $2 \pi$ ). A real phase value of $(-\pi)$ could be equal in a complex representation to $(-\pi+2 \pi=+\pi)$. Thus, the phase jumps from $(-\pi)$ to $(+\pi)$. In order to avoid the filtering of phase jumps, before the unwrapping process, it is more convenient to consider the complex domain for the interferometric phase noise reduction, where phase jumps are not present. Moreover, the interferometric phase is represented as a given point on the unit circle. Lets consider

$$
e^{j \varphi_{z}}=\cos \left(\varphi_{z}\right)+j \sin \left(\varphi_{z}\right)
$$

Then, the complex InSAR phase noise model for (10) could be derived easily from (9) for each term (real and imaginary part). Then, the complex interferometric phase noise model is given by:

$$
\left\{\begin{array}{l}
\cos \left(\varphi_{z}\right)=\cos \left(\varphi_{x}\right) \underbrace{\cos (v)}_{v_{1}}-\sin \left(\varphi_{x}\right) \underbrace{\sin (v)}_{v_{2}} \\
\sin \left(\varphi_{z}\right)=v_{1} \sin \left(\varphi_{x}\right)+v_{2} \cos \left(\varphi_{x}\right)
\end{array}\right.
$$

where the original phase $\varphi_{x}$ is separated from the noise contribution $v$. In (López-Martínez \& Fabregas, 2002), Lopez and Fabregas had proved and validated this model using simulated and real interferometric phases. They deduced a more practical complex model given by:

$$
\left\{\begin{array}{l}
\cos \left(\varphi_{z}\right)=N_{c} \cos \left(\varphi_{x}\right)+v_{c} \\
\sin \left(\varphi_{z}\right)=N_{c} \sin \left(\varphi_{x}\right)+v_{s}
\end{array}\right.
$$

where $N_{c}$ is the mean value of the noise contribution $v_{1}$, which can be represented by the addition of its mean plus zero-mean random variable $\left(v_{1}^{\prime}\right)$

$$
v_{1}=\cos (v)=N_{c}+v_{1}^{\prime}
$$

The same representation can be used for the noise contribution $v_{2}$

$$
v_{2}=\sin (v)=N_{s}+v_{2}^{\prime}
$$

where $N_{s}=0$ (López-Martínez \& Fabregas, 2002) is the mean value of $v_{2}$ and $\left(v_{2}^{\prime}\right)$ the zeromean random variable. Thus, the expressions of $v_{c}$ and $v_{s}$ are given by:

$$
\left\{\begin{array}{l}
v_{c}=v_{1}^{\prime} \cos \left(\varphi_{x}\right)-v_{2}^{\prime} \sin \left(\varphi_{x}\right) \\
v_{s}=v_{1}^{\prime} \sin \left(\varphi_{x}\right)+v_{2}^{\prime} \cos \left(\varphi_{x}\right)
\end{array}\right.
$$

\section{InSAR phase noise reduction using a multiresolution approach}

The multi-look process reduces some noise. The remaining noise has to be reduced by a filtering process such as the adaptive Suksmono filtering (Suksmono \& Hirose, 2002), adaptive Lee filtering (Lee et al., 1998) or Goldstein-Werner (G-W) filtering (Goldstein \& Werner, 1998). In this chapter, we consider a multiresolution filtering approach.

\subsection{Multiresolution image filtering in the wavelet domain}

The wavelet transform (WT) decomposes a signal into its low frequency components (approximations or residues) and high frequency components (details) (Mallat, 1998). The resultant set of Coefficients is a pyramidal multiresolution representation of the original signal. The most approximated component is located at the lowest resolution level and other levels 
consist of corresponding detail components. The original signal is recovered in the reconstruction process by synthesizing these approximated and detail components. In the discrete wavelet transform (DWT), decomposition and reconstruction are conducted by filtering processes, called the wavelet filter banks. Ideal filters for the DWT are needed in order to preserve the linear characteristics of the WT. The ideal assumption holds if the DWT is computed using the Shannon wavelet (Vidakovic, 1999).

Filtering in the wavelet domain allows one to perform signal processing operations that are localized in both space and frequency (Hess-Nielsen \& Wickerhauser, 1996). This can be advantageous in many non-stationary problems, such as radar imaging (Odegard et al., 1995) and particularly SAR interferometry (Zhou et al., 1999). Furthermore, empirical evidence shows that wavelet bases generally provide more efficient representations of real-world data than pixel or frequency domain representations. Because wavelets are able to concisely represent complicated signal structure, filtering techniques based on the wavelet decomposition are much better at separating signals from noise than classical approaches based in the pixel or frequency domain. From a theoretical perspective, it has been shown that, because wavelet bases are unconditional bases for wide classes of signals, the wavelet transform is optimal for noise reduction (Donoho \& Johnstone, 1995).

In this chapter we consider the InSAR phase noise reduction in the wavelet domain. This choice is argued by the facts that:

- Filtering the InSAR phase noise is necessary for increasing the precision of practical measurements (extracted from the InSAR phase topography). Thus, The practical application of InSAR requires that the position of the fringes in the interferogram can't be shifted after being filtered.

- The fringe pattern in the interferogram have to be preserved after the filtering process. The localization of the wavelet functions is particularly to preserve fine details like edges (Donoho \& Johnstone, 1995).

- Filtering in the wavelet domain don't need a windowing process such for the classical filters. Indeed, these filters are based on a windowing process of the original signal. Consequently, the filter performance depends highly on the window dimensions.

\subsection{A complex wavelet model for InSAR phase filtering}

Using the above mentioned interferometric complex phase noise model ( $\S 2.3)$, Lopez and Fabregas proposed in (López-Martínez \& Fabregas, 2002) an equivalent model adapted in the wavelet domain. Assuming ideal filters for the two dimensions DWT $\left(\mathrm{DWT}_{2 D}\right)$, the noise model equations, for a complex interferometric phase image in the wavelet domain, have the following expressions (López-Martínez \& Fabregas, 2002):

$$
\left\{\begin{array}{l}
\mathrm{DWT}_{2 D}\left(\cos \left(\phi_{z}\right)\right)=2^{i} N_{c} \cos \left(\phi_{x}^{w}\right)+v_{c}^{w} . \\
\mathrm{DWT}_{2 D}\left(\sin \left(\phi_{z}\right)\right)=2^{i} N_{c} \sin \left(\phi_{x}^{w}\right)+v_{s}^{w}
\end{array}\right.
$$

where $v_{c}^{w}$ and $v_{s}^{w}$ represent the DWT of the noise terms $v_{c}$ and $v_{s}$, respectively, at the scale $2^{i} . \phi_{x}^{w}$ represents the phase information within the complex wavelet domain. The main characteristics of this model, as stated and proved in (López-Martínez \& Fabregas, 2002), can be summarized as follows:

- Each time a wavelet scale is calculated, the DWT increases the original signal component by a factor 2, without varying the power's noise. Indeed, the variance of the noise 
component in the wavelet domain has the same value as its variance in the spatial domain $\left(\sigma_{v_{v}^{w}}^{2}=\sigma_{v_{c}}^{2}\right.$ and $\left.\sigma_{v_{s}^{w}}^{2}=\sigma_{v_{s}}^{2}\right)$. This will allow a larger signal improvement for each new wavelet decomposition level.

- The wavelet coefficient intensity, computed using the defined real and imaginary parts, is directly related to the mean value of the noise contribution $v_{1}, N_{c}$. The mean of the intensity for a wavelet coefficient has the expression

$$
E\left\{\left|\operatorname{DWT}_{2 D}\left(e^{j \phi_{z}}\right)\right|^{2}\right\}=2^{2 i} N_{c}^{2}+\sigma_{v_{c}^{w}}^{2}+\sigma_{v_{s}^{w}}^{2}
$$

When the wavelet coefficient contains only noise (i.e., $N_{c}=0$, as $N_{c}$ is monotone increasing with the coherence), its value is reduced to $\left(\sigma_{v_{c}^{w}}^{2}+\sigma_{v_{c}^{v}}^{2}\right)$. However, for $N_{c} \neq 0$, and considering three wavelet decomposition levels, Lopez and Fabregas (López-Martínez \& Fabregas, 2002) show that, $\sigma_{v_{c}^{w}}^{2}+\sigma_{v_{s}^{w}}^{2}$ is negligible compared with $2^{6} N_{c}$, and then

$$
E\left\{\left|\operatorname{DWT}_{2 D}\left(e^{j \phi_{z}}\right)\right|^{2}\right\} \simeq 2^{2 i} N_{c}^{2}
$$

- For the phase of the wavelet coefficients $\phi_{z}^{w}$, it has the following expression

$$
\arg \left[\operatorname{DWT}_{2 D}\left(e^{j \phi_{z}}\right)\right]=\operatorname{arctang}\left(\frac{2^{i} N_{c} \sin \left(\phi_{x}^{w}\right)+v_{s}^{w}}{2^{i} N_{c} \cos \left(\phi_{x}^{w}\right)+v_{c}^{w}}\right)
$$

Where, $\arg []$, represents the argument of a complex quantity. When the wavelet coefficient contains only noise, its phase is given by

$$
\arg \left[\operatorname{DWT}_{2 D}\left(e^{j \phi_{z}}\right)\right]=\operatorname{arctang}\left(\frac{v_{s}^{w}}{v_{c}^{w}}\right)
$$

Moreover, when the wavelet coefficients are computed for a large number of decomposition levels $(\geq 3)$, and for $N_{c} \neq 0$, the interferometric wavelet phase is given as

$$
\arg \left[\operatorname{DWT}_{2 D}\left(e^{j \phi_{z}}\right)\right]=\operatorname{arctang}\left(\frac{2^{i} N_{c} \sin \left(\phi_{x}^{w}\right)}{2^{i} N_{c} \cos \left(\phi_{x}^{w}\right)}\right) \simeq \phi_{x}^{w}
$$

Thus, we deduce the original (without noise) wrapped interferometric phase.

Considering the above properties, of the presented interferometric noise model in the wavelet domain, Lopez and Fabregas had developed a noise reduction algorithm, which flow diagram is illustrated in Fig. 2. The used filtering algorithm is then based on the analysis of the wavelet residue bands (signal bands) using the discret packet transform and it is composed of six steps. In the following, we resume these steps considering the paper of Lopez and Fabregas (LópezMartínez \& Fabregas, 2002).

- Step 1: A three wavelet scales transform is applied to the complex interferometric phase. In the third decomposition level, the wavelet filter is applied to all the bands (signals $\left(\mathrm{a}_{2}\right)+$ details of the second decomposition level $\left.\left(\mathrm{d}_{2}\right)\right)$, using the Discrete Wavelet Packet Transform (DWPT). This is in order to obtain a constant amplification by a factor $2^{3}$ of the signal wavelet coefficient intensities of the first decomposition level. A detailed description of the nomenclature of the DWPT is depicted in Fig. 3. 


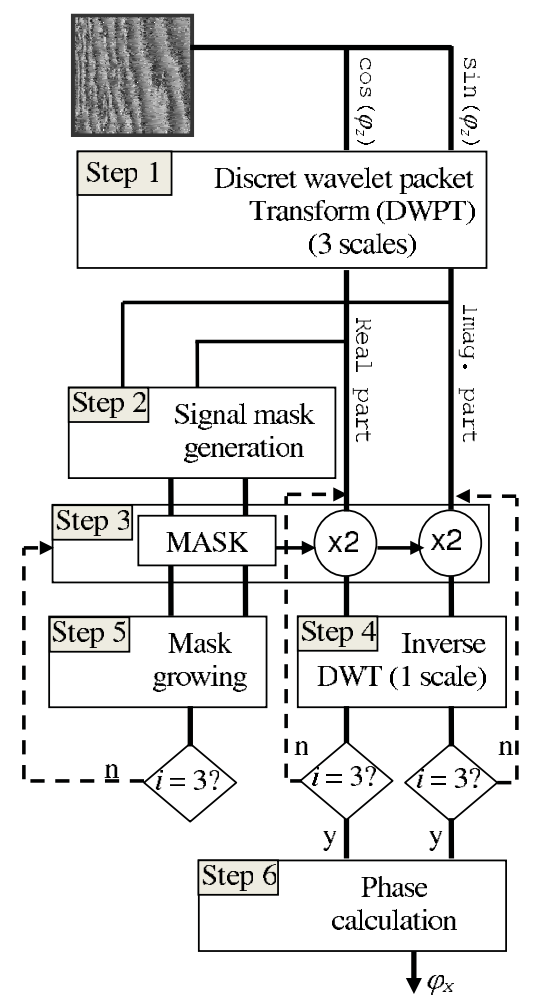

Fig. 2. Noise reduction algorithm flow diagram, proposed by Lopez and Fabregas (LópezMartínez \& Fabregas, 2002).

- Step 2: A mask, indicating the pixel locations of the signal coefficients is generated. This is done using a two defined parameters: The signal quality $\left(\Gamma_{\text {sig }}\right)$ and the threshold $\left(T h_{w}\right)$ parameter. $\left(\Gamma_{\text {sig }}\right)$ is given by the following expression

$$
\Gamma_{\mathrm{sig}}=\frac{I_{w}-2^{2 i} \sigma_{w}^{2}}{I_{w}}
$$

where $I_{w}$ represents the intensity for those wavelet coefficients in the low frequency sub-band ( $\mathrm{a}_{2}$ and all the $\mathrm{d}_{2}$ ) and $\sigma_{w}^{2}$ is the noise variance in the corresponding spatial area described by $I_{w}$ in the noise bands $\left(\mathrm{d}_{1}\right)$. To detect the wavelet signal coefficients, a threshold $\left(t h_{w}\right)$ is applied to $\Gamma_{\text {sig }}$

$$
\begin{aligned}
& \Gamma_{s i g} \geq t h_{w} \Rightarrow \text { Signal coefficient } \\
& \Gamma_{s i g}<t h_{w} \Rightarrow \text { Noise coefficient. }
\end{aligned}
$$

The threshold value considered by Lopez and Fabregas in (López-Martínez \& Fabregas, 2002) is equal for all the 16 signal bands. It defines up to which coherence level the 


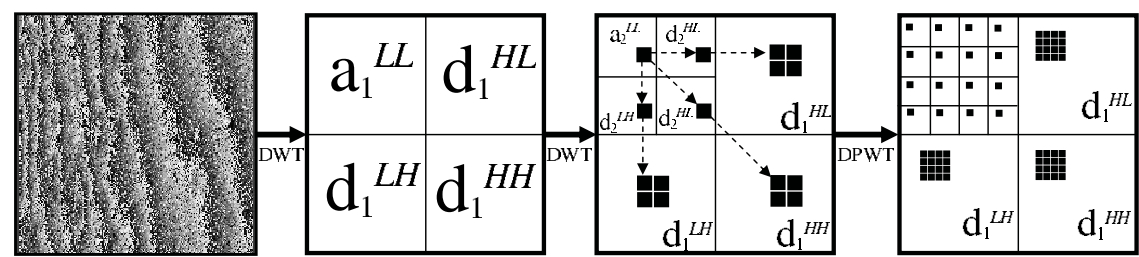

Fig. 3. Wavelet transformation process employed to transform interferometric complex phase images. Relations between pixels (black boxes) in different scales are shown (López-Martínez \& Fabregas, 2002).

signal is processed. In the resulting mask, those isolated coefficients that are detected as signal coefficients are removed in order to reduce noise effects in the mask.

- Step 3: The real and imaginary parts of those coefficients detected as signal in the previous step are multiplied by two. For the noise coefficients the real and imaginary parts are maintained.

- Step 4: The inverse DWT is applied but only reducing one wavelet scale.

- Step 5: To obtain a mask locating signal coefficients for previous scales (higher frequency scales), a new mask is derived from the one generated in Step 2. Each four bands in a scale $2^{i}$ are derived from a single band in the previous wavelet scale $2^{i-1}$, where a 1-to-4 space relation is established between wavelet coefficients. First, the masks of the four bands at the scale $2^{i}$ are merged through a logical OR operation. Then, the dimensions of the merged mask are doubled to fit the $2^{i-1}$ scale band dimensions. In this case, if a pixel of the merged mask is classified as signal, the four pixels referring to the same spatial area, but in the band of the scale $2^{i-1}$, are also classified as signal; otherwise they are classified as noise. This sequence of mask growing allows to obtain a mask locating useful signals in the original domain.

Then, we iterate (dashed lines in Fig. 2) the sequence of Steps 3-5 three times in order to obtain the complex signal in the original domain.

- Step 6: From the complex output of the algorithm, the phase is calculated, which is the estimation of the original interferometric phase.

\subsection{The proposed filtering algorithm}

We propose in this section a modified version of the Lopez and Fabregas algorithm for the SAR interferometric phase noise reduction in the wavelet domain. We aim by this modification to overcome the inconsistencies in the resulting filtered interferogram caused by the extrapolation in the mask growing step. Compared to this method, our proposal explained below has two different features:

- The inverse discret wavelet transform (DWT) is applied considering an adaptive mask extracted from the InSAR coherence map, sub-sampled to the convenient resolution of the corresponding wavelet decomposition level (to be processed).

- The threshold is adaptive and it is computed based on the equivalent 4 signal bands, and not all the signal bands. 


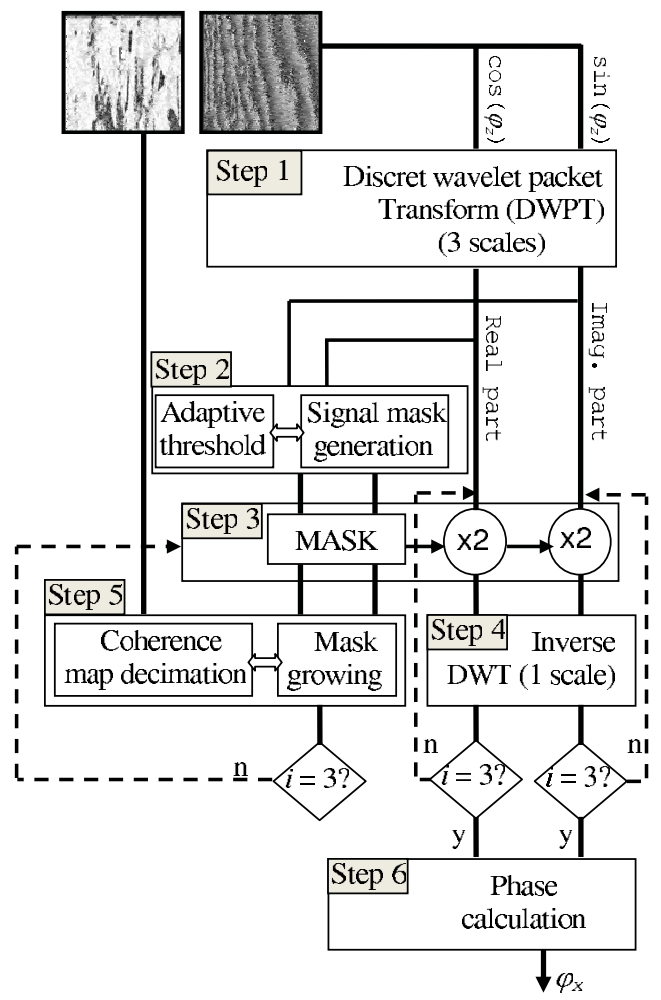

Fig. 4. Noise reduction algorithm flow diagram, proposed by Lopez and Fabregas (LópezMartínez \& Fabregas, 2002).

Figure 4 is the flow diagram of the developed algorithm named FAMM (for Filtrage par Approche Multi-échelle Modifiée). Both algorithms, the FAMM and the Lopez ones, consist of the same six steps. However, the processing in Step 2 and Step 5 is different. In fact:

- In Step 2, when generating the mask, the Lopez algorithm consider the same threshold for all the 16 ( 4 sub-bands from $\mathrm{a}_{2}$ and $4^{*} 3$ sub-bands from $\mathrm{d}_{2}$ ) signal bands, where we do not. The reason is that the $a_{2}$ and the $d_{2}$ are not the resulting coefficients of the same process, and so they don't have necessary the same signal dynamic. This could be confirmed in (Buccigrossi \& Simoncelli, 2001), where we find that the $\mathrm{d}_{2}$ sub-bands have a generalized gaussian distribution (GGD) and the $\mathrm{a}_{2}$ coefficient has not. Thus, we define two thresholds, $t h_{w a}$ and $t h_{w d}$, for each sub-band category. The values of these thresholds are computed with respect to the mean of the sub-band dynamics.

- In Step 5, when doubling the dimensions of the merged mask to fit the $2^{i-1}$ scale band dimensions, we do not systematically classify the four pixels, in the band of the scale $2^{i-1}$, to the same class. We propose to generate a sub-sampled coherence map, from the initial InSAR coherence one, having the same dimensions as the the band of the scale 
$2^{i-1}$. Then, the mask growing will depend on the coherence values of the four considered pixels in the band of the scale $2^{i-1}$. Figure 5 shows an illustrative example of the mask growing step for a given $(m, n)$ pixel in the band of the scale $2^{i}$ and its corresponding four pixels in the band of the scale $2^{i-1},\{(k, l) ;(k, l+1) ;(k+1, l) ;(k+1, l+1)\}$. The decision rule will depend on:

1. If the pixel $(m, n)$ in the mask is a signal coefficient, so

$$
\begin{array}{r}
\left|\rho(k, l)-\rho_{p}\right| \leq \varepsilon_{\mathcal{C}} \Rightarrow p \text { is a signal coefficient } \\
\left|\rho(k, l)-\rho_{p}\right|>\varepsilon_{\mathcal{C}} \Rightarrow p \text { is a noise coefficient }
\end{array}
$$

where $p \in\{(k, l+1) ;(k+1, l) ;(k+1, l+1)\}$

2. If the pixel $(m, n)$ in the mask is a noise coefficient, so

$$
\begin{aligned}
& \left|\rho(k, l)-\rho_{p}\right| \leq \varepsilon_{\mathcal{c}} \Rightarrow p \text { is a noise coefficient } \\
& \left|\rho(k, l)-\rho_{p}\right|>\varepsilon_{\mathcal{C}} \Rightarrow p \text { is a signal coefficient }
\end{aligned}
$$

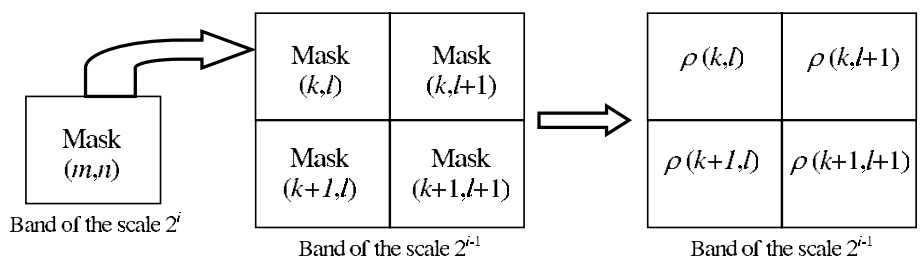

Fig. 5. Mask growing taking into account, the InSAR coherence map.

\subsection{Noise reduction on simulated and real interferograms}

In order to validate the proposed noise reduction algorithm, synthetic and real interferograms were used. The accuracy of the denoising process was estimated using two different approaches:

- The PSNR, computed between the filtered and the original simulated interferograms. It is given by:

$$
P S N R=10 \log _{10}\left(\frac{(2 \pi)^{2}}{M S E}\right)
$$

where MSE is the Mean Square Error between the filtered and the original simulated interferograms.

- The difference image distribution, computed between the filtered and the original real interferograms.

Moreover, a quantitative comparison (using the PSNR ratio) with alternative interferometric phase filters addressed in the literature is reported. 
Table 1. Interferometric phase filtering PSNR for the cone and pyramid interferograms, with noise variance of $10^{-2}$.

\begin{tabular}{ccccc}
\hline \hline & Interferogram & $\begin{array}{c}\text { Gaussian } \\
\text { noise }(\mathrm{dB})\end{array}$ & $\begin{array}{c}\text { White } \\
\text { noise }(\mathrm{dB})\end{array}$ & $\begin{array}{c}\text { Uniform } \\
\text { noise }(\mathrm{dB})\end{array}$ \\
\hline Lopez & cone & 39.9 & 39.85 & 41.5 \\
(López-Martínez \& Fabregas, 2002) & pyramid & 39.51 & 39.77 & 40.89 \\
\hline FAMM & cone & 41.36 & 41.29 & 42.78 \\
& pyramid & 41.13 & 40.81 & 41.67 \\
\hline Lee & cone & 37.18 & 37.52 & 37.22 \\
(Lee \& Liu, 1999) & pyramid & 36.74 & 37.03 & 37.28 \\
\hline
\end{tabular}

The simulated data were a 512x512 pixel interferograms, representing a cone and a pyramid, relatives to single look complexe images, with coherence varying from 0.9 to 0.45 . More details could be found in (Abdelfattah \& Bouzid, 2008) about the simulation process. The experimental results given in (Abdelfattah \& Bouzid, 2008) show that the Daubechies 20-coefficient wavelet transform gives the best PSNR for both cone and pyramid interferograms. Table 1 shows the numerical comparison for these simulated interferograms. The used empirical parameters for the thresholds were $t h_{w a}=-0.4, t h_{w d}=-0.2$ and $\varepsilon_{c}=6.10^{-4}$. The results in Table 1 confirm that the FAMM filtering algorithm allows a gain more than $2 \mathrm{~dB}$ compared to the one of Lopez and Fabregas (López-Martínez \& Fabregas, 2002) and more than 4 dB compared to the Lee one (Lee \& Liu, 1999).

In order to test the spatial resolution maintenance properties of the different above mentioned filters, we simulated a pyramid with phase noise coherence equal to 0.45 and a ten-pixel fringe period. The critical point is to maintain the pyramid edges. Fig. 6 shows the noisy phase image and the result obtained after applying the different filters. The pyramid edges are clearly maintained for both the FAMM and the Lopez Filters.

We also tested the developed noise reduction algorithm (FAMM) on a real experimental ERS2 interferogram of Capbon, in the north of Tunisia. This single look complex interferometric phase has a 2048×2048 pixel dimension with an approximate spatial resolution of $25 \times 25 \mathrm{~m}$. This phase image has been filtered by the FAMM filter using the same parameters as those used for the simulated interferograms. From Fig. 9 (c), one can notice that the algorithm, like the Lopez one (López-Martínez \& Fabregas, 2002), is able to process, in the same efficient way, areas with smooth or steep slopes at the same time. Figure 9 (d) shows the difference between the original and the filtered phases, which has a mean equal to zero and does not contain any image detail, demonstrating that the proposed filter preserves the topographic information.

\section{InSAR phase unwrapping based on MRF model}

The aim of this section is to present a general framework for the phase unwrapping problem, based on Markov random fields (MRF(s)) models (Griffeath, 1976). The proposed algorithm exploit Bayesian estimation theory to perform energy minimization in order to reconstruct the original phase field. Our contribution consists in the exploitation of the Interferometric phase distribution (Abdelfattah \& Nicolas, 2006) in order to develop an adaptive energy functional for the optimization of the MRF to be retrieved. 


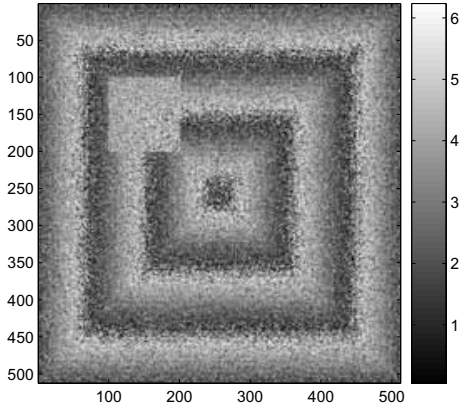

(a)

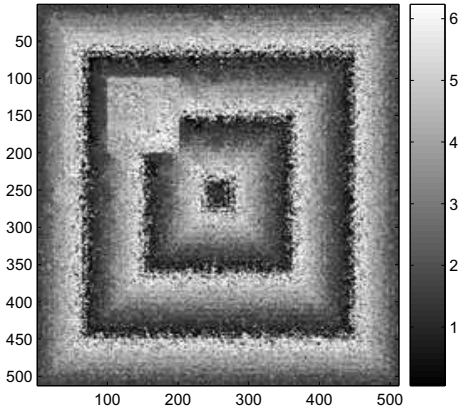

(c)

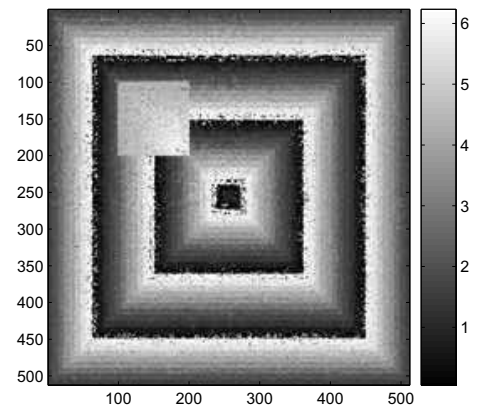

(b)

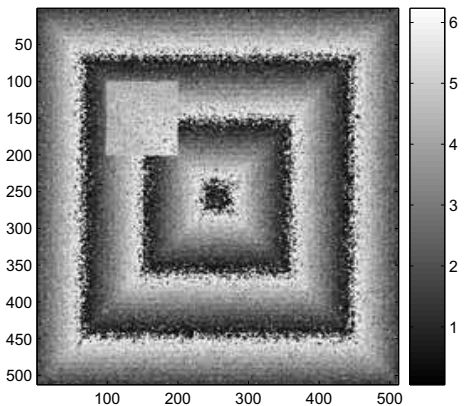

(d)

Fig. 6. Interferometric phase filtering results with an interferogram representing a pyramid with $|\rho|=0.45$ and a variance Gaussian noise $10^{-2}$. (a) Original noisy image, (b) FAMM filter, (c) Lopez filter and (d) Lee filter.

\subsection{General context}

The problem of recovering the absolute phase field from observations is ill-posed and can be solved if one first regularizes it by introducing further information (prior knowledge) about the behavior of the solution. To introduce this additional information, we exploit a statistical framework, which consist in considering that the searched field $\phi$ is a Markov random one (Griffeath, 1976). Thanks to the Hammersly-Clifford theorem (Griffeath, 1976), which establishes a correspondence between MRF and Gibbs distribution, the distribution of $\phi$ field is expressed with the following representation (Griffeath, 1976):

$$
P(\phi)=\frac{1}{Z} \exp (-U(\phi))
$$

where $Z$ is a normalizing constant, and $U(\phi)$ is a function which encodes the local characteristics of the phase behavior being modelled. The true phase field $\phi$ is related to the wrapped phase field $\varphi$ through the equation given by:

$$
\phi=\varphi+2 k \pi
$$




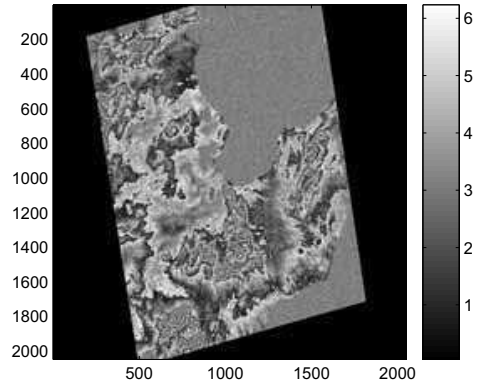

(a)

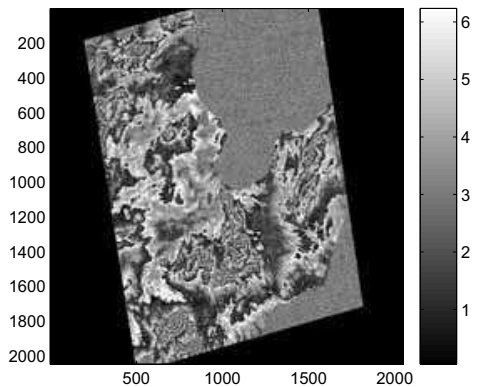

(c)

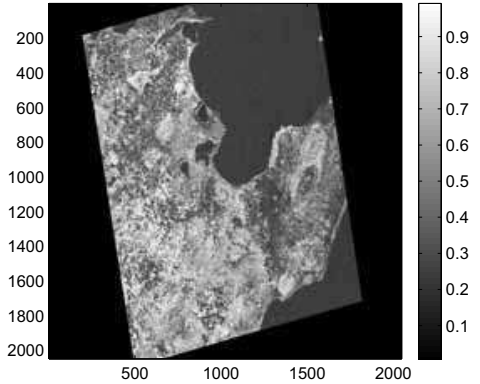

(b)

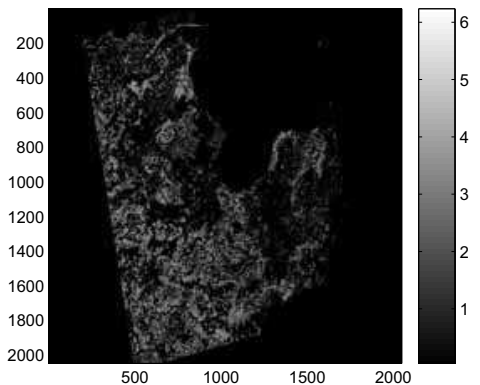

(d)

Fig. 7. Filtering result with the FAMM filter of (a) the original interferometric phase of Capbon (ERS2 interferogram), (b) the corresponding coherence map, (c) The filtered interferogram and (d) the difference image (original interferogram - filtered interferogram).

where $k$ is the integer to determine for each $(i, j)$ pixel in the $\varphi$ field, in order to solve (24) and recover $\phi$. Considering the MRF model in two dimensional processing, each pixel $(i, j)$ of an image is defined as a site $s$ in a set of regular lattice of nodes $S$. Let us assume that the observed data obey to the equation, related to each site $s$ given by (Rodriguez-Vera \& Servin, 1995):

$$
\phi_{s}=\varphi_{s}+2 \pi k_{s}+q
$$

where $\phi_{s}$ the phase to be retrieved at the site $s, \varphi_{s}$ is the observed phase at the site $s$ and $q$ is a constant. We introduce a new field $f$ as a correction field, defined by the elements $f_{s}$, where $s \in S$ and given by (Rodriguez-Vera \& Servin, 1995):

$$
f_{s}=k_{s}+\frac{q}{2 \pi}
$$

Note that the values of each element $f_{s}$ of the field $f$, are not necessarily integers $\left(f_{s} \in \mathbb{R}\right)$, but the differences are, so that for any two neighboring sites $\{s, t\} \in S$, we have (Rodriguez-Vera \& Servin, 1995):

$$
f_{s}-f_{t}=r\left(f_{s}-f_{t}\right)
$$


where $f_{s}$ and $f_{t}$ are phase values related to two neighboring sites and $r(x)$ is the closet integer to $x$. Thus, (25) yields (Rodriguez-Vera \& Servin, 1995):

$$
\phi_{s}=\varphi_{s}+2 \pi f_{s}
$$

Assuming (28), that introduce the correction field $f$, is verified everywhere in the lattice structure of the phase field $\phi$, then we obtain the conditional probability, $P(\varphi, f \mid \phi)$, of the observed phase $\varphi$ and $f$ given $\phi$, that takes the form (Rodriguez-Vera \& Servin, 1995):

$$
P(\varphi, f \mid \phi)=\left\{\begin{array}{cc}
1 & \text { if } \\
0 & \text { otherwise }
\end{array} \quad \phi_{s}=\varphi_{s}+2 \pi f_{s} \quad \forall s \in S\right.
$$

we note that the likelihood distribution $P(\varphi, f \mid \phi)$ corresponds to Dirac impulse response, and can be expressed by (Rodriguez-Vera \& Servin, 1995):

$$
P(\varphi, f \mid \phi)=\frac{1}{Z_{1}} \exp \left(-\alpha \sum_{s \in S}\left[\varphi_{s}-\phi_{s}+2 \pi f_{s}\right]^{2}\right)
$$

where $Z_{1}$ is a normalizing constant and $\alpha$ is a weight parameter.

\subsection{Prior and posterior model}

To define a probabilistic model that describes the real interferometric phase information, it is necessary to define an energy functional:

$$
U(\phi)=\sum_{c \in C \mid s \in c} U_{c}(\phi)
$$

where the $U_{\mathcal{c}}($.$) are called potentials functions, indexed by the clique c$ in the set of cliques $C$ of the neighborhood system of a given site $s$. Note that each potential $U_{c}$ depends only on the values taken on the clique sites $x_{s}, s \in c$, and, therefore, accounts only for local interactions between neighboring pixels. As consequence, local dependencies in the field $\phi$ can be modelled by defining suitable potentials. In the literature, a number of models have been proposed for various applications, for further comprehension refer to ( $\mathrm{Li}, 2001)$. To enforce global smoothness of reconstructed phase, a quadratic potential model could be adopted (Rodriguez-Vera \& Servin, 1995):

$$
U_{c}(\phi)=\left(\phi_{s}-\phi_{t}\right)^{2}
$$

The equations (31) and (32) gives:

$$
U(\phi)=\sum_{c \in C \mid s \in c}\left(\phi_{s}-\phi_{t}\right)^{2}
$$

Probabilistic description of the current estimate of the field $(\phi, f)$, given the observed data $\varphi$, is calculated using Bayes'rule (Rodriguez-Vera \& Servin, 1995):

$$
P(\phi, f \mid \varphi)=\frac{P(\varphi \mid \phi, f) P(\phi, f)}{P(\varphi)}
$$

Assuming that $P(\varphi)$ is a constant, and that the correction field $f$ obey to the prior model defined by (23), we obtain:

$$
\begin{aligned}
P(\phi, f \mid \varphi) & \propto P(\varphi \mid \phi, f) P(\phi, f) \\
& \propto \frac{1}{Z_{2}} \exp (-U(\phi, f))
\end{aligned}
$$


where $Z_{2}$ is a normalizing constant, and $U(\phi, f)$ is the posterior energy function, given by merging (23), (30) and (14) (Rodriguez-Vera \& Servin, 1995):

$$
U(\phi, f)=\sum_{c \in C \mid s \in c} U_{c}(\phi)+\alpha \sum_{s \in S}\left(\varphi_{s}-\phi_{s}+2 \pi f_{s}\right)^{2}
$$

The maximum a posteriori (MAP) estimator for $\phi$ may now be obtained by the minimization of (37). Using (28), and requiring that $\left[f_{s}-f_{t}-r\left(f_{s}-f_{t}\right)\right]^{2}$ be small for nearest-neighbor pairs of sites, the minimization problem involving two unknown fields $\phi$ and $f$, may be simplified by expressing the energy in term of the field $f$ only. This allows to absorb the noise and interpolate the missing data. The new expression of the posterior energy becomes (RodriguezVera \& Servin, 1995):

$$
U(f)=\sum_{c \in C \mid s \in \mathcal{c}} U_{c}(\varphi+2 \pi f)+\alpha \sum_{t \in V_{s}}\left[f_{s}-f_{t}-r\left(f_{s}-f_{t}\right)\right]^{2}
$$

where $V_{s}$ is a set of sites neighbouring the site $s$ and $\alpha$ is a regularization parameter that controls the smoothness of $f$.

\subsection{Minimization algorithm-Gradient descent method}

The gradient descent optimization method could be adopted to restor the field $f$ that minimize the energy function denoted by (38), and hence to unwrap $\varphi$. this iterative method is useful to prevent the initialization problem related to the application of the MRF models for phase unwrapping procedure (Rodriguez-Vera \& Servin, 1995). to start the decent algorithm with the appropriate initial state, we must set the initial value as follow:

$$
f_{s}=-\frac{\varphi_{s}}{2 \pi}
$$

The implementation of the minimization algorithm, in which the gradient descent method is performed by updating the value of each variable $f_{s}$ of the field $f$, in parallel programming way, according to the following equation (Rodriguez-Vera \& Servin, 1995):

$$
f_{s}^{(n+1)}=f_{s}^{(n)}-h \frac{\partial U\left[f^{(n)}\right]}{\partial f_{s}}
$$

where $n$ denotes the iteration number, and $h$ is the step size of the gradient descent. The gradient of $U$ is expressed by (Rodriguez-Vera \& Servin, 1995):

$$
\begin{aligned}
\frac{\partial U\left(f^{(n)}\right)}{\partial f_{s}} & =\sum_{c \in C \mid s \in c} \frac{\partial U_{c}\left(\varphi+2 \pi f^{(n)}\right)}{\partial f_{s}} \\
& +2 \lambda \sum_{t \in V_{s}}\left[f_{s}^{(n)}-f_{t}^{(n)}-r\left(f_{s}^{(n)}-f_{t}^{(n)}\right)\right]
\end{aligned}
$$

For the regular lattice $S$, we consider the first-order system, so we have $C=C_{1} \cup C_{2}$, with $C_{1}=\{s \mid s \in S\}$ and $C_{2}=\left\{\{s, t\} \mid t \in V_{s}, s \in S\right\}$. Using (32) the developed form of (40) and then the gradient descent automaton is given by (Rodriguez-Vera \& Servin, 1995):

$$
\begin{aligned}
f_{s}^{(n+1)} & =f_{s}^{(n)}-2 h\left[\sum_{t \in V_{s}}\left\{2 \pi\left(g_{s}-g_{t}\right)+4 \pi^{2}\left(f_{s}^{(n)}-f_{t}^{(n)}\right)\right\}\right. \\
& \left.+\lambda\left(f_{s}^{(n)}-f_{t}^{(n)}-r\left(f_{s}^{(n)}-f_{t}^{(n)}\right)\right)\right]
\end{aligned}
$$


where $V_{s}=t:\|s-t\|=1$ and where we set $\varphi_{s}=0$ and $f_{s}=0 \forall s \notin S$.

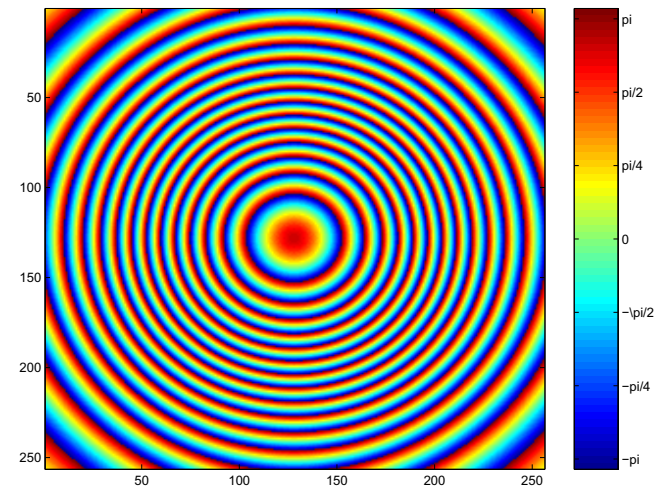

(a)

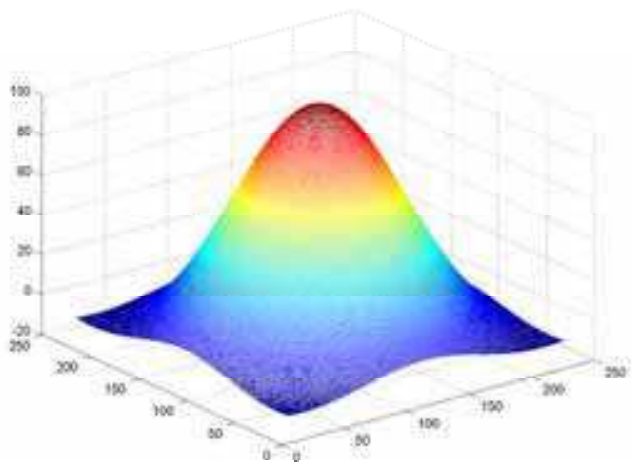

(b)

Fig. 8. (a) Interferogram generated from a Gaussian DEM with a maximum height of $100 \mathrm{~m}$ and standard deviations (in rows $\left(\sigma_{x}\right)$ and columns $\left(\sigma_{y}\right)$ ) of $\sigma_{x}=\sigma_{y}=2.10^{-4}$. (b) Reconstructed elevation data obtained with the gradient descent algorithm..

Figure 8 displays the interferogram generated from a synthetic Gaussian DEM with a maximum height of $100 \mathrm{~m}$ and standard deviations (in rows $\left(\sigma_{x}\right)$ and columns $\left(\sigma_{y}\right)$ ) of $\sigma_{x}=\sigma_{y}=$ $2.10^{-4}$. The algorithm described above works well with highly coherent interferograms (for data affected by low noise level). However, gradient descent method does not give satisfactory results in the case of noisy interferograms. In fact, the chosen a priori model does not integrate a noise model. Thus, it is necessary to accommodate the potential function to recover the discontinuities (noise) present in the observed data. A modification of the prior potentials is proposed in (Rodriguez-Vera \& Servin, 1995), making the phase jumps, that overcomes a certain threshold $a$, contribute with a fixed amount to the energy, independently of their size. A truncated quadratic potentials, are then proposed and the (32) is replaced by (Rodriguez-Vera \& Servin, 1995):

$$
U_{c}(\phi)=\left\{\begin{array}{lc}
\left(\phi_{s}-\phi_{t}\right)^{2} & \text { if } \\
a^{2} & \text { otherwise }
\end{array}\left|\phi_{s}-\phi_{t}\right|<a,\right.
$$

where $a$ is a positive parameter.

Figure 9 (a) illustrates a noisy (mean $=0.6$ and standard deviation $=0.9$ ) interferogram generated from a two nearby Gaussians elevation data of height $100 \mathrm{~m}$ and $50 \mathrm{~m}$, with local inconsistencies (nul data) marked by blue squares. However, as we can see on Fig. 9 (b), the reconstructed elevation data obtained with the gradient descent algorithm is not perfect, in spite of the correction of the inconsistencies. This have lead us to think for an adaptive energy functional modelling.

\subsection{Our contribution}

In order to enforce the global smoothness of the algorithm, the above considered quadratic potentials is given by:

$$
V_{i, j}\left(z_{i}, z_{j}\right)=\left(z_{i}-z_{j}\right)^{2}
$$




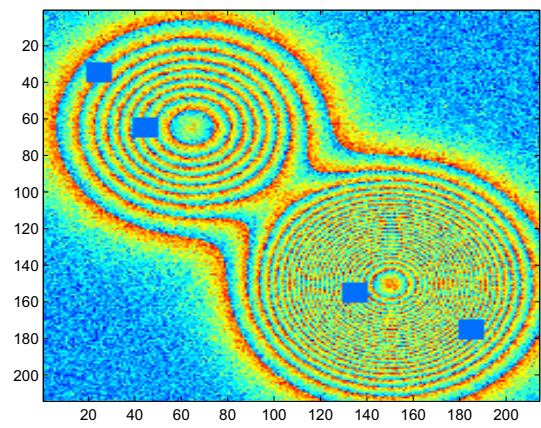

(a)

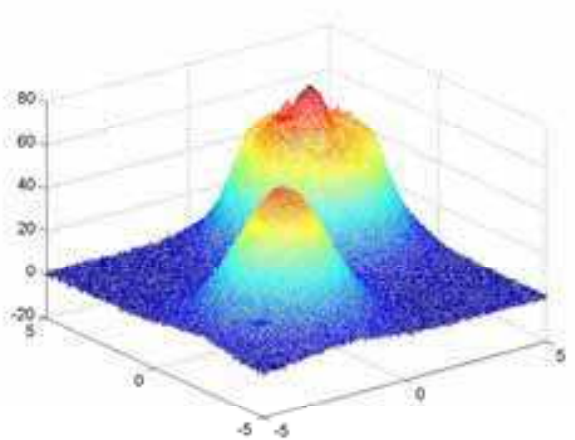

(b)

Fig. 9. (a) Interferogram generated from a two nearby Gaussians elevation data of height 100 $\mathrm{m}$ and $50 \mathrm{~m}$, with local inconsistencies marked by blue squares. (b) Reconstructed elevation data obtained with the gradient descent algorithm..

Such a potential function makes this algorithm work well for smooth surface. However it fails to recover discontinuous surfaces as well. Our main contribution in this work is to propose a modification to the above prior potential so that the jumps contribution to the energy are mitigated by weighting them by the probability of their occurrence which decrease when they increase, the proposed potential function is then equivalent to (Elmzoughi et al., 2008):

$$
V_{i, j}\left(z_{i}, z_{j}\right)=\left(z_{i}-z_{j}\right)^{2} P_{Z}\left(z_{i}-z_{j}\right) .
$$

In our case, $Z$ corresponds to the absolute phase $\phi$ which pdf can be written as an expression implying hypergeometric functions. However, A good approximation can be given by a Gaussian model:

$$
P_{\Phi}(\phi)=\frac{1}{\sqrt{2 \pi} \sigma_{\phi}} \exp \left(-\frac{(\phi-\widehat{\phi})^{2}}{\sigma_{\phi}^{2}}\right),
$$

where $\sigma_{\phi}$ and $\widehat{\phi}$ are respectively the variance and the mean of the absolute phase $\phi$. Theses parameters can be easily estimated from the theoretical hypergeometric pdf. By considering the transfert theorem, the gradient descent automation in equation (38) could be given by:

$$
\begin{aligned}
f_{i}^{t+1}= & f_{i}^{t}-\frac{\sqrt{2 \pi} h}{\sigma_{\phi}} \times \sum_{j \in V_{i}}\left[\left[g_{i}-g_{j}+2 \pi\left(f_{i}^{t}-f_{j}^{t}\right)\right] \exp \frac{\left[g_{i}-g_{j}+2 \pi\left(f_{i}^{t}-f_{j}^{t}\right)\right]^{2}}{4 \sigma_{\phi}^{2}}\right], \\
& \times\left[1-\frac{1}{4 \sigma_{\phi}^{2}}\left[g_{i}-g_{j}+2 \pi\left(f_{i}^{t}-f_{j}^{t}\right)\right]\right]+\lambda\left[f_{i}^{t}-f_{j}^{t}-r\left(f_{i}^{t}-f_{j}^{t}\right)\right]
\end{aligned}
$$

where $V_{i}$ is the set of neighbors of the considered site $i$. In (Elmzoughi et al., 2008), it is shown that both algorithms seems to be equivalent for smooth surfaces. However, using the proposed potential function allowed to ameliorate results, especially in terms of mean square errot and in terms of SNR, for surfaces with discontinuities. This could be explained by a better preservation of these discontinuities in the reconstructed phase. 


\section{Conclusion}

In this chapter, we proposed a modified filtering algorithm to the López and Fabregas (LópezMartínez \& Fabregas, 2002) noise reduction algorithm for the interferometric phase noise in SAR interferometry using a multiresolution approach. Our contribution to the existing algorithm consists on the exploitation of the InSAR coherence map in order to generate a more adaptive mask for each decomposition level.

Moreover, we presented a general probabilistic framework to phase unwrapping problem based on the work of Rodriguez and Servin (Rodriguez-Vera \& Servin, 1995). Both MRF and Bayesian estimator were applied to recover the desired phase, as the optimal field solution of the maximum a posteriori (MAP) estimation criterion. An iterative method that minimizes a general energy function is proposed, and a parallel algorithm based on gradient descent optimization is designed to perform this task. The proposed solution overcomes some important limitations of most of the phase unwrapping procedures, and the results show robustness, and stability.

These results give us new ideas for the applications of the InSAR unwrapping phase MRF algorithm for unwrapping interferometric synthetic Aperture Sonar (InSAS) (Bonifant et al., 2000) phase. Both two phases present similar statistics. However, if the ground truth could be used for the InSAR result validations, it not the case for the InSAS results. In fact, the ability to produce full coverage bathymetric maps and generate accurate measurements of the seafloor height, is limited. So, an adaptive unwrapping procedure could be interesting.

\section{References}

Abdelfattah, R. \& Bouzid, A. (2008). Sar interferogram filtering in the wavelet domain using a coherence map mask, Proceedings of the 15th IEEE International Conference on Image Processing, ICIP 2008, San Diego, CA, pp. 1888-1891.

Abdelfattah, R. \& Nicolas, J. (2002). Topographic sar interferometry formulation for highprecision dem generation, IEEE Transaction on Geoscience and Remote Sensing Vol. 40(No. 11): pp. 2415-2426.

Abdelfattah, R. \& Nicolas, J. (2006). Interferometric sar coherence magnitude estimation using second kind statistics, IEEE Transaction on Geoscience and Remote Sensing Vol. 44(No. 7): pp. 1942-1953.

Balzter, H. (2001). Forest mapping and monitoring with interferometric synthetic aperture radar (insar), Progress in physical geography Vol. 25(No. 2): pp. 159-177.

Bodart, C., Gassani, J., Salmon, M. \& Ozer, A. (2009). Contribution of sar interferometry (from ers1/2) in the study of aeolian transport processes: The cases of niger, mauritania and morocco, in A. Marini \& M. Talbi (eds), Desertification and Risk Analysis Using High and Medium Resolution Satellite Data Training Workshop on Mapping Desertification, Springer, pp. 129-136.

Bonifant, W., Richards, M. \& McClellan, J. (2000). Interferometric height estimation of the seafloor via synthetic aperture sonar in the presence of motion errors, IEE Proceedings - Radar, Sonar and Navigation Vol. 147(No. 6): pp. 322-330.

Buccigrossi, R. \& Simoncelli, E. (2001). Image compression via joint statistical characterization in the wavelet domain, IEEE Transactions on Image Processing Vol. 8(No. 12): pp. 16881701. 
Donoho, D. \& Johnstone, I. (1995). The $z \pi m$ algorithm: a method for interferometric image reconstruction in sar/sas, Journal of the American Statistical Association Vol. 90(No. 432): pp. 1200-1224.

Elmzoughi, A., Maalej, A., Abdelfattah, R. \& Belhadj, Z. (2008). Insar phase unwrapping based on a combination of markov random fields and hypergeometric phase pdf models, Proceedings of the IEEE International Geoscience and Remote Sensing Symposium, IGARSS 2008, Boston, Massachusetts, pp. 538-541.

Franceschetti, G. \& Lanari, R. (1999). Synthetic Aperture Radar processing, CRC Press.

Gabriel, A. \& Goldstein, R. (1988). Retrieval of vegetation parameters with sar interferometry, International Journal of Remote Sensing Vol. 9(No. 4): pp. 857-872.

Ghiglia, D. \& Pritt, M. (1998). Two-dimensional phase unwrapping: Theory, algorithms and software, John Wiley \& Sons Inc.

Ghiglia, D. \& Romero, L. (1994). Robust two-dimensional weighted and unweighted phase unwrapping that uses fast transforms and iterative methods, Journal of the Optical Society of America A Vol. 11(No. 1): pp. 107-117.

Goldstein, R. \& Werner, C. (1998). Radar interferogram filtering for geophysical applications, Geophysical Research Letters Vol. 25(No. 21): pp. 4035-4038.

Goldstein, R., Zebker, H. \& Werner, C. (1988). Satellite radar interferometry: two-dimensional phase unwrapping, Radio Science Vol. 23(No. 4): pp. 713-720.

Graham, L. (1974). Synthetic interferometer radar for topographic mapping, Proceedings of the IEEE Vol. 62(No. 6): pp. 763-768.

Griffeath, D. (1976). Introduction to markov random fields, in J. Kemeny, J. Snell \& A. Knapp (eds), Denumerable Markov Chains, Springer-Verlag, pp. 424-457.

Hanssen, R. (2001). Radar Interferometry: Data Interpretation and Error Analysis, Kluwer Academic Publishers.

Hess-Nielsen, N. \& Wickerhauser, M. (1996). Wavelets and time frequency analysis, Proceedings of the IEEE Vol. 82(No. 4): pp. 523-540.

Lee, H. \& Liu, J. G. (1999). Spatial decorrelation due to topography in the interferometric sar coherence imagery, Proceedings of the IEEE International Geoscience and Remote Sensing Symposium IGARSS'99, Hamburg, Germany, pp. 485-487.

Lee, J., Hoppel, W. \& Mango, S. (1994). Intensity and phase statistics of multilook polarimetric and interferometric sar imagery, IEEE Transaction on Geoscience and Remote Sensing Vol. 32(No. 5): pp. 1017-1027.

Lee, J., Papathanassiou, K., Ainsworth, T., Grunes, M. \& Reigber, A. (1998). A new technique for phase noise filtering of sar interferometric phase images, IEEE Transaction on Geoscience and Remote Sensing Vol. 36(No. 5): pp. 1456-1465.

Li, S. (2001). Markov Random Field Modeling in Image Analysis, Springer-Verlag.

López-Martínez, C. \& Fabregas, X. (2002). Modeling and reduction of sar interferometric phase noise in the wavelet domain, IEEE Transaction on Geoscience and Remote Sensing Vol. 40(No. 12): pp. 2553-2566.

Maître, H. (2001). Traitement des images de RSO, Hermes.

Mallat, S. (1998). A wavelet tour of signal processing, Academic Press.

Massonet, D. \& Feigl, K. (1998). Radar interferometry and its application to changes in the earth's surface, Reviews of Geophysics Vol. 36(No. 4): pp. 441-500.

Mattar, K., Vachon, P., Geudtner, D., Gray, A., Cumming, I. \& Brugman, M. (1998). Validation of alpine glacier velocity measurements using ers tandem-mission sar data, IEEE Transaction on Geoscience and Remote Sensing Vol. 36(No. 3): pp. 974-984. 
Odegard, J., Guo, H., Lang, M., Burrus, C., Jr., R. . W., Novak, L. \& Hiett, M. (1995). Wavelet based sar speckle reduction and image compression, Proceedings of the SPIE Symposium on OE/Aerospace Sensing and Dual Use Photonics, Orlando, Florida, pp. 17-21.

Rodriguez-Vera, R. \& Servin, M. (1995). Modeling and reduction of sar interferometric phase noise in the wavelet domain, Journal of the Optical Society of America A Vol. 12(No. 12): pp. 2578-2585.

Suksmono, A. \& Hirose, A. (2002). Adaptive noise reduction of insar images based on a complex-valued mrf model and its application to phase unwrapping problem, IEEE Transaction on Geoscience and Remote Sensing Vol. 40(No. 3): pp. 699-709.

Touzi, R. \& Lopez, A. (1996). Statistics of the stokes parametersand of the complex coherence parameters in one-look and multilook speckle fields, IEEE Transaction on Geoscience and Remote Sensing Vol. 34(No. 2): pp. 519-531.

Vidakovic, B. (1999). Statistical Modeling by Wavelets, Wiley-interscience.

Wegmullerl, U. \& Werner, C. (1997). Retrieval of vegetation parameters with sar interferometry, IEEE Transaction on Geoscience and Remote Sensing Vol. 35(No. 1): pp. 18-24.

Wishart, J. (1928). The generalized product moment distribution in samples from a normal multivariate population, Biometrika Vol. 20A(No. 1/2): pp. 32-52.

Zebker, H. \& Villasenor, J. (1992). Decorrelation in interferometric radar echoes, IEEE Transaction on Geoscience and Remote Sensing Vol. 30(No. 5): pp. 950-959.

Zhou, Y., Li, D. \& Genderen, J. V. (1999). Wavelet based sar speckle reduction and image compression, Proceedings of the SPIE Symposium Vol. 3723, Wavelet Applications VI, Orlando, Florida, pp. 208-214. 
Advances in Geoscience and Remole Sensing

Advances in Geoscience and Remote Sensing

Edited by Gary Jedlovec

ISBN 978-953-307-005-6

Hard cover, 742 pages

Publisher InTech

Published online 01, October, 2009

Published in print edition October, 2009

Remote sensing is the acquisition of information of an object or phenomenon, by the use of either recording or real-time sensing device(s), that is not in physical or intimate contact with the object (such as by way of aircraft, spacecraft, satellite, buoy, or ship). In practice, remote sensing is the stand-off collection through the use of a variety of devices for gathering information on a given object or area. Human existence is dependent on our ability to understand, utilize, manage and maintain the environment we live in - Geoscience is the science that seeks to achieve these goals. This book is a collection of contributions from world-class scientists, engineers and educators engaged in the fields of geoscience and remote sensing.

\section{How to reference}

In order to correctly reference this scholarly work, feel free to copy and paste the following:

Riadh Abdelfattah (2009). InSAR phase analysis: Phase unwrapping for noisy SAR interferograms, Advances in Geoscience and Remote Sensing, Gary Jedlovec (Ed.), ISBN: 978-953-307-005-6, InTech, Available from: http://www.intechopen.com/books/advances-in-geoscience-and-remote-sensing/insar-phase-analysis-phaseunwrapping-for-noisy-sar-interferograms

\section{INTECH}

open science | open minds

\section{InTech Europe}

University Campus STeP Ri

Slavka Krautzeka 83/A

51000 Rijeka, Croatia

Phone: +385 (51) 770447

Fax: +385 (51) 686166

www.intechopen.com

\section{InTech China}

Unit 405, Office Block, Hotel Equatorial Shanghai

No.65, Yan An Road (West), Shanghai, 200040, China

中国上海市延安西路65号上海国际贵都大饭店办公楼405单元

Phone: +86-21-62489820

Fax: +86-21-62489821 
(C) 2009 The Author(s). Licensee IntechOpen. This chapter is distributed under the terms of the Creative Commons Attribution-NonCommercial-ShareAlike-3.0 License, which permits use, distribution and reproduction for non-commercial purposes, provided the original is properly cited and derivative works building on this content are distributed under the same license. 